\title{
Finite-Time Stability of Continuous Time Delay Systems: Lyapunov-like Approach with Jensen's and Coppel's Inequality
}

\author{
Dragutin Lj. Debeljkovic $^{(1)}$, Sreten B. Stojanovic ${ }^{(2)}$, \\ Aleksandra M. Jovanovic ${ }^{(1)}$ \\ ${ }^{(1)}$ Faculty of Mechanical Engineering, University of Belgrade, Department of \\ Control Engineering, 11000 Belgrade, Serbia, e-mail: ddebeljkovic@mas.bg.ac.rs \\ ${ }^{(2)}$ Faculty of Technology, University of Nis, Department of Engineering Sciences \\ and Mathematics, 16000 Leskovac, Serbia, e-mail: sstojanovic@tf.ni.ac.rs
}

Abstract: In this paper, the finite-time stability (FTS) of linear continuous time-delay systems is studied. By using suitable Lyapunov-like function and Jensen's and Coppel's inequality, a FTS condition is derived as a set of algebraic inequalities. The comparison of this method with some previous one is done and it has been showed that the numerical computation is reduced.

Keywords: time delay systems; finite-time stability; continuous systems; Jensen's integral inequality; Coppel's inequality

\section{Introduction}

Asymptotic stability, BIBO stability and other classical stability concepts deal with systems operating over an infinite time interval. However, in many practical cases, larger values of the state variables are not allowable in the specified (finite) time interval. Then, instead of asymptotic stability, it is preferable to use the stability defined over a finite time interval, i.e. finite-time stability (FTS). A system will be FTS if its state does not exceed some previously defined limit, for a given time interval. This concept stability dates back to the 1950s [1-3]. In references [4-11] some controllers are proposed such that the feedback system is FTS.

Many technical systems, such as pneumatic, hydraulic and electric systems, as well as process systems in the chemical industry, possess time-delay. The stability analysis of time-delay systems is more complex because the time-delay impairs the system stability. Similar to the non-delay systems, we can define FTS for time- 
delay systems. In references [12-18], some basic results on FTS are derived. These results are conservative, because they use the inequalities based on the norm of state vector. Recently, using new boundary technique based on the vector and matrix inequality, integral or no integral type, some less conservative results are obtained [19-22].

This article considers a novel delay-dependent FTS sufficient condition of linear continuous time-delay systems. The combination of Lyapunov-like approach and two algebraic inequalities (Jensen's integral inequality and Coppel's inequality) is used to solve this stability problem. The condition is expressed in the form of a set of algebraic inequality.

Notation. $\mathfrak{R}^{n}$ and $\mathfrak{R}^{n \times m}$ denote the $n$-dimensional Euclidean space and set of all $n \times m$ real matrices. $X>0$ means that $X$ is real positive definite symmetric matrix; $X>Y$ is equivalent to $X-Y>0 . \mu(X)=1 / 2 \lambda_{\max }\left(X+X^{T}\right)$ is matrix measure of matrix $X$.

\section{Preliminaries and Problem Formulation}

Consider the following linear time-delay system:

$$
\dot{x}(t)=A_{0} x(t)+A_{1} x(t-\tau)
$$

with a initial conditions:

$$
x(t)=\phi(t), \quad t \in[-\tau, 0]
$$

where $x(t) \in \mathfrak{R}^{n}$ is the state vector, $A_{0} \in \mathfrak{R}^{n \times n}, A_{1} \in \mathfrak{R}^{n \times n}$ and $B \in \mathfrak{R}^{n \times m}$ are constant matrices and $\tau$ is time-delay.

In the process of derivation of the stability condition, following definition and three lemmas are used.

Definition 1. [22] Time-delay system (1) satisfying the given initial condition (2) is said to be finite-time stable (FTS) with respect to $(\alpha, \beta, T)$ if

$$
\sup _{t \in[-\tau, 0]} \phi^{T}(t) \phi(t) \leq \alpha \Rightarrow x^{T}(t) x(t)<\beta, \forall t \in[0, T]
$$

Lemma 1. [22] (Jensen's integral inequality) For any positive symmetric constant matrix $M \in \square^{n \times n}$, scalars $a, b$ satisfying $a<b$, a vector function $f:[a, b] \rightarrow \square^{n}$ such that the integrations concerned are well defined, then: 


$$
\left(\int_{a}^{b} f(\theta) d \theta\right)^{T} M\left(\int_{a}^{b} f(\theta) d \theta\right) \leq(b-a) \int_{a}^{b} f^{T}(\theta) M f(\theta) d \theta
$$

Lemma 2. [12] (Coppel's inequality) For any real square matrix $M \in \square^{n \times n}$ and scalar variable $t$, the following expression holds:

$$
\lambda_{\max }\left(e^{M t} \cdot e^{M^{T} t}\right) \leq e^{2 \mu(M) t}
$$

where $\mu(M)$ is matrix measure of the matrix $M$.

Lemma 3. For any symmetric positive definite matrix $\Gamma=\Gamma^{T}>0$, the following expressions hold:

$$
\begin{aligned}
& 2 u(t) v(t) \leq u^{T}(t) \Gamma u(t)+v^{T}(t) \Gamma^{-1} v(t) \\
& -2 u(t) v(t) \leq u^{T}(t) \Gamma u(t)+v^{T}(t) \Gamma^{-1} v(t)
\end{aligned}
$$

\section{Main Result}

In this section Lyapunov-like approach will be used in order to find sufficient delay dependent FTS conditions of the time-delay system (1). The following lemma, that is necessary for the design of Lyapunov-like function, is developed. We note that the new result is based on the result given in [23].

Lemma 4. Let a scalar function $V(y(t))$ be defined by:

$$
V(y(t))=y^{T}(t) y(t)
$$

where $y(t)$ is vector which is defined by:

$$
y(t)=x(t)+\int_{0}^{\tau} Q(\theta) x(t-\theta) d \theta
$$

$x(t) \in \mathfrak{R}^{n}$ is the state vector of the system (1), $Q(t) \in \mathfrak{R}^{n \times n}$ is continuous and differentiable matrix function over time interval $[0, \tau]$ satisfying the following differential matrix equation:

$$
\dot{Q}(\vartheta)=\left(A_{0}+Q(0)\right) Q(\vartheta), \quad \vartheta \in[0, \tau]
$$

with initial condition: 


$$
Q(\tau)=A_{1}
$$

Then, derivative of $V(y(t))$ is given with:

$$
\dot{V}(y(t))=y^{T}(t) \Xi y(t)
$$

where:

$$
\Xi=\left(A_{0}+Q(0)\right)^{T}+\left(A_{0}+Q(0)\right)
$$

Proof. From (8), follows:

$$
\begin{aligned}
\dot{V}(y(t)) & =\left(\dot{x}^{T}(t)+\frac{d}{d t} \int_{0}^{\tau} x^{T}(t-\theta) Q^{T}(\theta) d \theta\right) \times\left(x(t)+\int_{0}^{\tau} Q(\eta) x(t-\eta) d \eta\right) \\
& +\left(x^{T}(t)+\int_{0}^{\tau} x^{T}(t-\theta) Q^{T}(\theta) d \theta\right) \times\left(\dot{x}(t)+\frac{d}{d t} \int_{0}^{\tau} Q(\eta) x(t-\eta) d \eta\right)
\end{aligned}
$$

First derivative of the integral term $\int_{0}^{\tau} Q(\theta) x(t-\theta) d \theta$ can be determined as follows. From

$$
\begin{aligned}
& \frac{d}{d \theta}(Q(\theta) x(t-\theta))=\dot{Q}(\theta) x(t-\theta)+Q(\theta) \frac{\partial}{\partial \theta}(x(t-\theta)) \\
& \frac{\partial}{\partial \theta}(x(t-\theta))=-\frac{\partial}{\partial t}(x(t-\theta))
\end{aligned}
$$

we get:

$$
\frac{d}{d \theta}(Q(\theta) x(t-\theta))=\dot{Q}(\theta) x(t-\theta)-Q(\theta) \frac{\partial}{\partial t}(x(t-\theta))
$$

or rearranging:

$$
Q(\theta) \frac{\partial}{\partial t}(x(t-\theta))=\dot{Q}(\theta) x(t-\theta)-\frac{d}{d \theta}(Q(\theta) x(t-\theta))
$$

Using the following identity:

$$
\frac{d}{d t}(Q(\theta) x(t-\theta))=Q(\theta) \frac{\partial}{\partial t}(x(t-\theta))
$$

one can finally have: 


$$
\begin{aligned}
\frac{d}{d t} \int_{0}^{\tau} Q(\theta) x(t-\theta) d \theta & =\int_{0}^{\tau} \dot{Q}(\theta) x(t-\theta) d \theta-\frac{d}{d \theta} \int_{0}^{\tau} Q(\theta) x(t-\theta) d \theta \\
& =\int_{0}^{\tau} \dot{Q}(\theta) x(t-\theta) d \theta-Q(\tau) x(t-\tau)+Q(0) x(t)
\end{aligned}
$$

Employing (11), we have:

$$
\frac{d}{d t} \int_{0}^{\tau} Q(\theta) x(t-\theta) d \theta=\int_{0}^{\tau} \dot{Q}(\theta) x(t-\theta) d \theta-A_{1} x(t-\tau)+Q(0) x(t)
$$

Finally, (14) becomes:

$$
\begin{aligned}
\dot{V} & (y(t))= \\
& =\left(\begin{array}{l}
x^{T}(t) A_{0}^{T}+x^{T}(t-\tau) A_{1}^{T}+ \\
\left.+\int_{0}^{\tau} x^{T}(t-\theta) \dot{Q}^{T}(\theta) d \theta-x^{T}(t-\tau) A_{1}^{T}+x^{T}(t) Q^{T}(0)\right) \times
\end{array}\right. \\
& \times\left(x(t)+\int_{0}^{\tau} Q(\eta) x(t-\eta) d \eta\right) \\
& \left(x^{T}(t)+\int_{0}^{\tau} x^{T}(t-\theta) Q^{T}(\theta) d \theta\right) \times \\
& \times\left(\begin{array}{c}
A_{0} x(t)+A_{1} x(t-\tau)+ \\
\left.+\int_{0}^{\tau} \dot{Q}(\eta) x(t-\eta) d \eta-A_{1} x(t-\tau)+Q(0) x(t)\right)
\end{array}\right.
\end{aligned}
$$

or:

$$
\begin{aligned}
\dot{V}(y(t)) & =\left(x^{T}(t) A_{0}^{T}+x^{T}(t) Q^{T}(0)+\int_{0}^{\tau} x^{T}(t-\theta) \dot{Q}^{T}(\theta) d \theta\right) \times \\
& \times\left(x(t)+\int_{0}^{\tau} Q(\eta) x(t-\eta) d \eta\right) \\
& +\left(x^{T}(t)+\int_{0}^{\tau} x^{T}(t-\theta) Q^{T}(\theta) d \theta\right) \times \\
& \times\left(A_{0} x(t)+Q(0) x(t)+\int_{0}^{\tau} \dot{Q}(\eta) x(t-\eta) d \eta\right)
\end{aligned}
$$

and, after some simple manipulations, follows: 


$$
\begin{aligned}
& \dot{V}(y(t))=x^{T}(t)\left(\left(A_{0}^{T}+Q^{T}(0)\right)+\left(A_{0}+Q(0)\right)\right) x(t) \\
& +x^{T}(t) \int_{0}^{\tau}\left(A_{0}^{T} Q(\eta)+Q^{T}(0) Q(\eta)+\dot{Q}(\eta)\right) x(t-\eta) d \eta \\
& \quad+\left(\int_{0}^{\tau} x^{T}(t-\theta)\left(Q^{T}(\theta) A_{0}+Q^{T}(\theta) Q(0)+\dot{Q}^{T}(\theta)\right) d \theta\right) x(t) \\
& \quad+\int_{0}^{\tau} \int_{0}^{\tau} x^{T}(t-\theta)\left(\dot{Q}^{T}(\theta) Q(\eta)+Q^{T}(\theta) \dot{Q}(\eta)\right) x(t-\eta) d \theta d \eta
\end{aligned}
$$

By the virtue of (10), one can get:

$$
\begin{aligned}
& \dot{V}(y(t))=x^{T}(t) \Xi x(t) \\
& +x^{T}(t) \int_{0}^{\tau}\left(A_{0}^{T}+Q^{T}(0)+A_{0}+Q(0)\right) Q(\eta) x(t-\eta) d \eta \\
& +\left(\int_{0}^{\tau} x^{T}(t-\theta) Q^{T}(\theta)\left(A_{0}^{T}+Q^{T}(0)+A_{0}+Q(0)\right) d \theta\right) x(t) \\
& \quad+\int_{0}^{\tau} \int_{0}^{\tau} x^{T}(t-\theta)\left\{\begin{array}{l}
Q^{T}(\theta)\left(A_{0}^{T}+Q^{T}(0)\right) Q(\eta)+ \\
+Q^{T}(\theta)\left(A_{0}+Q(0)\right) Q(\eta)
\end{array}\right\} x(t-\eta) d \theta d \eta
\end{aligned}
$$

that is:

$$
\begin{aligned}
\dot{V}(y(t)) & =x^{T}(t) \Xi x(t)+x^{T}(t) \Xi \int_{0}^{\tau} Q(\eta) x(t-\eta) d \eta \\
& +\left(\int_{0}^{\tau} x^{T}(t-\theta) Q^{T}(\theta) d \theta\right) \Xi x(t) \\
& +\int_{0}^{\tau} \int_{0}^{\tau} x^{T}(t-\theta)\left(Q^{T}(\theta) \Xi Q(\eta)\right) x(t-\eta) d \theta d \eta
\end{aligned}
$$

as well as:

$$
\begin{aligned}
& \dot{V}(y(t))=x^{T}(t) \Xi\left(x(t)+\int_{0}^{\tau} Q(\eta) x(t-\eta) d \eta\right) \\
& \quad+\left(\int_{0}^{\tau} x^{T}(t-\theta) Q^{T}(\theta) d \theta\right) \Xi\left(x(t)+\int_{0}^{\tau} Q(\eta) x(t-\eta) d \eta\right)
\end{aligned}
$$

and finally: 


$$
\begin{aligned}
& \dot{V}(y(t))=x^{T}(t) \Xi y(t)+\left(\int_{0}^{\tau} x^{T}(t-\theta) Q^{T}(\theta) d \theta\right) \Xi y(t) \\
& \dot{V}(y(t))=\left[x^{T}(t)+\int_{0}^{\tau} x^{T}(t-\theta) Q^{T}(\theta) d \theta\right] \Xi y(t) \\
& \dot{V}(y(t))=y^{T}(t) \Xi y(t)
\end{aligned}
$$

what completes the proof.

Previously derived result will be used to obtain the following stability condition.

Theorem 1. The time-delayed system (1)-(2) with is finite-time stable with respect to $\{\alpha, \beta, T\}$ if there exists a positive scalar $\wp$ such that:

$$
\begin{aligned}
& x^{T}(t-\vartheta) x(t-\vartheta)<q x^{T}(t) x(t) \\
& q>0, \quad \vartheta \in[-\tau, 0], \quad \forall t \in[0, T] \\
& (1+\tau)(1+\psi)\left(1-\wp \psi-\frac{q \tau}{\wp}\right)^{-1} e^{\lambda_{\max }(\Xi) T}<\frac{\beta}{\alpha} \\
& \wp \in\left(\max \left\{\wp_{1}, 0\right\}, \quad \wp_{2}\right), \\
& \wp_{1,2}=\frac{1 \pm \sqrt{1-4 \psi \tau q}}{2 \psi}, \quad 4 \psi \tau q<1
\end{aligned}
$$

where:

$$
\begin{aligned}
& R=A_{0}+Q(0) \\
& \Xi=R^{T}+R \\
& \psi=\lambda_{\max }\left(Q(0) Q^{T}(0)\right) \frac{e^{2 \mu_{2}(R) \tau}-1}{2 \mu(R)}
\end{aligned}
$$

$\mu_{2}(R)$ being matrix measure of matrix $R$ and $Q(0)$ is any solution of the following nonlinear transcendental matrix equation:

$$
e^{A_{0}+Q(0) \tau} Q(0)=A_{1}
$$

Proof. From (12) follows:

$$
\dot{V}(y(t))=y^{T}(t) \Xi y(t) \leq \lambda_{\max }(\Xi) V(y(t))
$$


Integrating (38) from 0 to $t$, with $t \in[0, T]$, we have:

$$
V(y(t))<e^{\lambda_{\max }(\Xi) \cdot t} \cdot V(0)
$$

From (8), one can find:

$$
\begin{aligned}
V(y(0)) & =x^{T}(0) x(0)+2 \int_{0}^{\tau} x^{T}(0) Q(\vartheta) x(-\vartheta) d \vartheta \\
& +\left[\int_{0}^{\tau} Q(\vartheta) x(-\vartheta) d \vartheta\right]^{T} \times \int_{0}^{\tau} Q(\vartheta) x(-\vartheta) d \vartheta
\end{aligned}
$$

Based on the known inequality (6), for $\Gamma=I$, one can get:

$$
\begin{aligned}
& V(y(0)) \leq x^{T}(0) x(0)+\int_{0}^{\tau} x^{T}(0) Q(\vartheta) Q^{T}(\vartheta) x(0) d \vartheta \\
& \quad+\int_{0}^{\tau} x^{T}(-\vartheta) x(-\vartheta) d \vartheta+\left[\int_{0}^{\tau} Q(\vartheta) x(-\vartheta) d \vartheta\right]^{T} \times \int_{0}^{\tau} Q(\vartheta) x(-\vartheta) d \vartheta
\end{aligned}
$$

Using Jensen's inequality (4), we get:

$$
\begin{aligned}
& V(y(0)) \leq x^{T}(0) x(0)+\int_{0}^{\tau} x^{T}(0) Q(\vartheta) Q^{T}(\vartheta) x(0) d \vartheta \\
& \quad+\int_{0}^{\tau} x^{T}(-\vartheta) x(-\vartheta) d \vartheta+\tau \int_{0}^{\tau} x^{T}(-\vartheta) Q^{T}(\vartheta) Q(\vartheta) x(-\vartheta) d \vartheta
\end{aligned}
$$

Introducing the general solution of (10), given with:

$$
Q(\vartheta)=e^{R \vartheta} Q(0), \quad \vartheta \in[0, \tau], \quad R=A_{0}+Q(0)
$$

and by substituting (43) into (42), the following inequalities are obtained:

$$
\begin{aligned}
& V(y(0)) \leq x^{T}(0) x(0)+\int_{0}^{\tau} x^{T}(0) e^{R \vartheta} Q(0) Q^{T}(0) e^{R^{T} \vartheta} x(0) d \vartheta \\
& +\int_{0}^{\tau} x^{T}(-\vartheta) x(-\vartheta) d \vartheta+\tau \int_{0}^{\tau} x^{T}(-\vartheta) Q^{T}(0) e^{R^{T} \vartheta} e^{R \vartheta} Q(0) x(-\vartheta) d \vartheta
\end{aligned}
$$




$$
\begin{aligned}
& V(y(0)) \leq x^{T}(0) x(0)+\lambda_{\max }\left(Q(0) Q^{T}(0)\right) \int_{0}^{\tau} \lambda_{\max }\left(e^{R \vartheta} e^{R^{T} \vartheta}\right) x^{T}(0) x(0) d \vartheta \\
& +\int_{0}^{\tau} x^{T}(-\vartheta) x(-\vartheta) d \vartheta+\tau \int_{0}^{\tau} \lambda_{\max }\left(e^{R \vartheta} e^{R^{T} \vartheta}\right) x^{T}(-\vartheta) Q^{T}(0) Q(0) x(-\vartheta) d \vartheta \\
& V(y(0)) \leq x^{T}(0) x(0)+x^{T}(0) x(0) \cdot \lambda_{\max }\left(Q(0) Q^{T}(0)\right) \int_{0}^{\tau} \lambda_{\max }\left(e^{R \vartheta} e^{R^{T} \vartheta}\right) d \vartheta \\
& +\int_{0}^{\tau} x^{T}(-\vartheta) x(-\vartheta) d \vartheta+\tau \lambda_{\max }\left(Q^{T}(0) Q(0)\right) \int_{0}^{\tau} \lambda_{\max }\left(e^{R \vartheta} e^{R^{T} \vartheta}\right) x^{T}(-\vartheta) d \vartheta
\end{aligned}
$$

Based on Definition 1, one can find:

$$
\begin{aligned}
V(y(0)) \leq & \alpha+\alpha \lambda_{\max }\left(Q(0) Q^{T}(0)\right) \int_{0}^{\tau} \lambda_{\max }\left(e^{R \vartheta} e^{R^{T} \vartheta}\right) d \vartheta \\
& +\alpha \tau+\alpha \tau \lambda_{\max }\left(Q^{T}(0) Q(0)\right) \int_{0}^{\tau} \lambda_{\max }\left(e^{R \vartheta} e^{R^{T} \vartheta}\right) d \vartheta
\end{aligned}
$$

From Coppell's inequality, Lemma 2, follows:

$$
V(y(0)) \leq \alpha(1+\tau)+\alpha(1+\tau) \lambda_{\max }\left(Q(0) Q^{T}(0)\right) \int_{0}^{\tau} e^{2 \mu(R) \vartheta} d \vartheta
$$

or:

$$
\begin{aligned}
V(y(0)) & \leq \alpha(1+\tau)\left(1+\left.\lambda_{\max }\left(Q(0) Q^{T}(0)\right) \frac{e^{2 \mu(R) \vartheta}}{2 \mu(R)}\right|_{\vartheta=0} ^{\vartheta=\tau}\right) \\
& =\alpha(1+\tau)\left(1+\lambda_{\max }\left(Q(0) Q^{T}(0)\right) \frac{e^{2 \mu(R) \tau}-1}{2 \mu(R)}\right)
\end{aligned}
$$

or finally:

$$
V(y(0)) \leq \alpha(1+\tau)(1+\psi)
$$

Based on (8)-(9), we have:

$$
x^{T}(t) x(t)+2 \int_{0}^{\tau} x^{T}(t) Q(\eta) x(t-\eta) d \eta<V(y(t))
$$

or: 


$$
x^{T}(t) x(t)<V(y(t))-2 \int_{0}^{\tau} x^{T}(t) Q(\eta) x(t-\eta) d \eta
$$

Let us find the right second term in inequality (52). By using the inequality (7) for $\Gamma=p I>0$ and by virtue of (31) and (43), one can find:

$$
\begin{aligned}
& -2 \int_{0}^{\tau} x^{T}(t) Q(\eta) x(t-\eta) d \eta \leq \\
& \leq \wp \int_{0}^{\tau} x^{T}(t) Q(\eta) Q^{T}(\eta) x(t) d \eta+\frac{1}{\wp} \int_{0}^{\tau} x^{T}(t-\eta) x(t-\eta) d \eta \\
& \quad<\wp \int_{0}^{\tau} x^{T}(t) e^{R \eta} Q(0) Q^{T}(0) e^{R^{T} \eta} x(t) d \eta+\frac{q}{\wp} \int_{0}^{\tau} x^{T}(t) x(t) d \eta \\
& \quad \leq \wp \lambda_{\max }\left(Q(0) Q^{T}(0)\right) \int_{0}^{\tau} x^{T}(t) e^{R \eta} e^{R^{T} \eta} x(t) d \eta+\frac{q}{\wp} x^{T}(t) x(t) \int_{0}^{\tau} d \eta
\end{aligned}
$$

or:

$$
\begin{aligned}
& -2 \int_{0}^{\tau} x^{T}(t) Q(\eta) x(t-\eta) d \eta \leq \\
& \quad \leq \wp \lambda_{\max }\left(Q(0) Q^{T}(0)\right) x^{T}(t) x(t) \int_{0}^{\tau} \lambda_{\max }\left(e^{R \eta} e^{R^{T} \eta}\right) d \eta+\frac{q \tau}{\wp} x^{T}(t) x(t) \\
& \quad<\wp \lambda_{\max }\left(Q(0) Q^{T}(0)\right) x^{T}(t) x(t) \int_{0}^{\tau} e^{2 \mu(R) \eta} d \eta+\frac{q \tau}{\wp} x^{T}(t) x(t) \\
& \left.\quad \wp \lambda_{\max }\left(Q(0) Q^{T}(0)\right) x^{T}(t) x(t) \frac{e^{2 \mu(R) \vartheta}}{2 \mu(R)}\right|_{\eta=0} ^{\eta=\tau}+\frac{q \tau}{\wp} x^{T}(t) x(t)
\end{aligned}
$$

or:

$$
\begin{aligned}
& -2 \int_{0}^{\tau} x^{T}(t) Q(\eta) x(t-\eta) d \eta< \\
& \quad<\wp \lambda_{\max }\left(Q(0) Q^{T}(0)\right) \frac{e^{2 \mu(R) \tau}-1}{2 \mu(R)} x^{T}(t) x(t)+\frac{q \tau}{\wp} x^{T}(t) x(t) \\
& \quad=\left(\wp \psi+\frac{q \tau}{\wp}\right) x^{T}(t) x(t)
\end{aligned}
$$


Thus, by using (39), (50), (52) and (55), leads to:

$$
\begin{gathered}
x^{T}(t) x(t)<V(y(t))+\left(\wp \psi+\frac{q \tau}{\wp}\right) x^{T}(t) x(t) \\
\leq e^{\lambda_{\max }(\Xi) t} V(y(0))+\left(\wp \psi+\frac{q \tau}{\wp}\right) x^{T}(t) x(t) \\
\leq \alpha(1+\tau)(1+\psi) e^{\lambda_{\max }(\Xi) t}+\left(\wp \psi+\frac{q \tau}{\wp}\right) x^{T}(t) x(t)
\end{gathered}
$$

and:

$$
\left(1-\wp \psi-\frac{q \tau}{\wp}\right) x^{T}(t) x(t)<\alpha(1+\tau)(1+\psi) e^{\lambda_{\max }(\Xi) t}, \quad \forall t \in[0, T]
$$

where:

$$
1-\wp \psi-\frac{q \tau}{\wp}>0
$$

Obviously, if the condition (33) holds, then the inequality (58) is satisfied.

Finally, from the above inequality and (32), we get:

$$
x^{T}(t) x(t)<\beta, \quad \forall t \in[0, T]
$$

Remark 1. For the derived stability criteria, an existence of solution of the nonlinear algebraic matrix equation (37) is a necessary condition. In other words, the equation (37) must have at least one solution with respect to $Q(0)$, in order to Theorem 1 can be generally applied.

\section{Numerical Example}

Example 1. Given a system of the form:

$$
\begin{aligned}
& \dot{x}(t)=A_{0} x(t)+A_{1} x(t-0.1) \\
& \phi(t)=\left[\begin{array}{lll}
1 & 1 & 1
\end{array}\right]^{T}, \quad t \in[-0.1,0] \\
& A_{0}=\left[\begin{array}{ccc}
-1.7 & 1.7 & 0 \\
1.3 & -1 & 0.7 \\
0.7 & 1 & -0.6
\end{array}\right], \quad A_{1}=\left[\begin{array}{ccc}
1.5 & -1.7 & 0.1 \\
-1.3 & 1.5 & -0.3 \\
-0.7 & 1 & 0.1
\end{array}\right]
\end{aligned}
$$


It is obvious that:

$$
\phi^{T}(t) \phi(t)=3=\alpha, \quad t \in[-\tau, 0]
$$

Figures 1-2 show the initial response $x(t)$ and the norm of state vector $x^{T}(t) x(t)$ of the system (60). Notice that the system (60) is not asymptotically stable. In addition, we determine upper bound of $T$ such that the system (60) is FTS with respect $\{\alpha, \beta, T\}$.

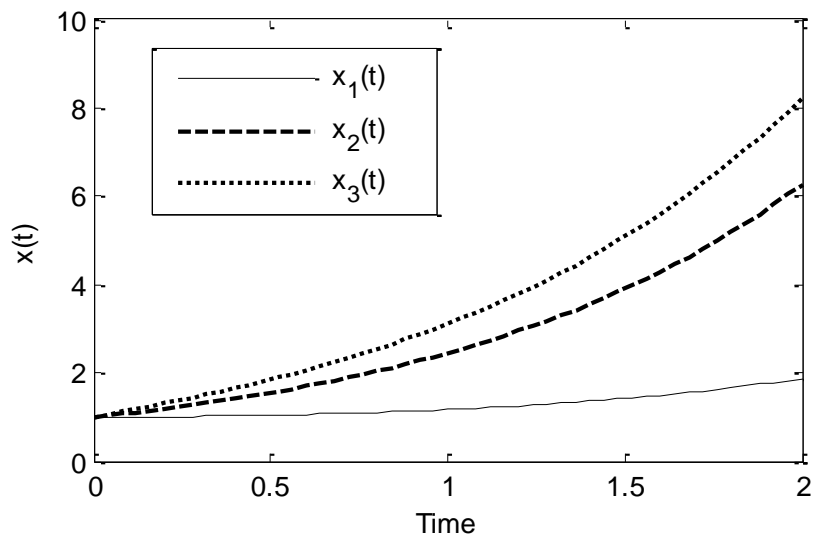

Figure 1

The state response $x(t)$ of the system (60)

Based on the initial response of the system (60), for following value of the parameter $q$ can be adopted so (31) is valid: $q=0.9$.

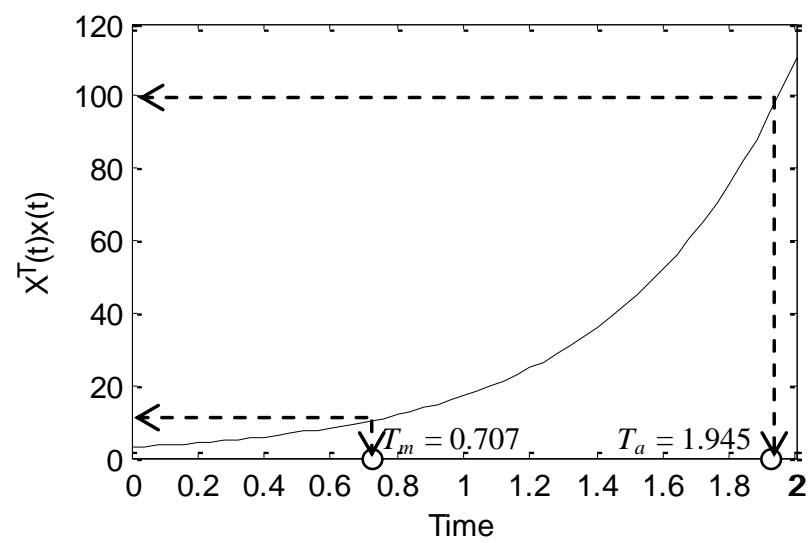

Figure 2

The norm $x^{T}(t) x(t)$ of the state vector of the system (60) 
From (34) and (37) one can find:

$$
\begin{aligned}
& Q(0)=\left[\begin{array}{ccc}
1.5279 & -1.7336 & 0.0994 \\
-1.2328 & 1.4145 & -0.2936 \\
-0.5249 & 0.8069 & 0.1575
\end{array}\right] \\
& R=\left[\begin{array}{ccc}
-0.1721 & -0.0336 & 0.0994 \\
0.0672 & 0.4145 & 0.4064 \\
0.1751 & 1.8069 & -0.4425
\end{array}\right], \Xi=\left[\begin{array}{ccc}
-0.3442 & 0.0335 & 0.2745 \\
0.0335 & 0.8290 & 2.2133 \\
0.2745 & 2.2133 & -0.8849
\end{array}\right]
\end{aligned}
$$

so:

$$
\begin{aligned}
& \lambda_{\max }\left\{Q(0) Q^{T}(0)\right\}=9.8109, \mu(R)=1.1789, \psi=1.1064, \\
& \wp_{1}=0.1014, \wp_{2}=0.8025, \wp \in(0.1014,0.8025), \\
& 4 \psi \tau q=0.3983<1, \lambda_{\max }\{\Xi\}=2.3578 .
\end{aligned}
$$

Let $\beta \in\{100,2000,5000\}$ and find upper bound of $T, T_{m}$, so the system (60) is FTS. The results of the stability analysis, for different values of the parameter $\beta$, are shown in Table 1 using various methods: [17], [18], [21] and Theorem 1 (this paper). The actual values of parameter $T, T_{a}$, are estimated from the norm of state vector and shown in Table 1 . Table 1 also lists the corresponding values of the parameter $\wp$.

Table 1

Upper bound of $T, T_{m}$

\begin{tabular}{|l|r|r|r|}
\hline & $\begin{array}{r}\beta=100, \\
\text { see Fig. 2 }\end{array}$ & $\begin{array}{r}\beta=2000, \\
T_{a}=3.525\end{array}$ & $\begin{array}{r}\beta=5000, \\
T_{a}=4.004\end{array}$ \\
\hline$[17]$ & 0.585 & 1.085 & 1.238 \\
\hline$[18]$ & 0.448 & 0.842 & 0.962 \\
\hline$[21]$ & 1.225 & 2.517 & 2.939 \\
\hline 22, without uncertainty $]$ & $\begin{array}{r}0.707, \text { see Fig. 2 } \\
(\wp=0.2865)\end{array}$ & $\begin{array}{r}1.978 \\
(\wp=0.2851)\end{array}$ & $\begin{array}{r}2.367 \\
(\wp=0.2837)\end{array}$ \\
\hline Theorem 1 &
\end{tabular}

From Table 1, it follows that Theorem 1 gives significantly better results than [17] and [18], but slightly poor results than [21] and [22]. However, unlike [21] and [22], which use LMI, Theorem 1 is based on algebraic inequalities, which can be solved without using appropriate optimization methods. Thus, compared to [21] and [22], the computational complexity of the presented stability criterion is significantly reduced. 


\section{Conclusion}

This paper considers FTS of linear continuous time-delay systems. The combination of Lyapunov-like approach and two algebraic inequalities (Jensen's and Coppel's inequality) is used. The new sufficient, delay-dependent FTS criterion with algebraic inequality has been derived. The obtained result reduces the numerical computation.

\section{Acknowledgment}

This work was supported in part by the Ministry of Science and Technological Development of Serbia under the Project OI 174001.

\section{References}

[1] Dorato P.: Short Time Stability in Linear Time-Varying System, Proc. IRE Internat. Conv. Rec. Part 4, New York, 1961, pp. 83-87

[2] Weiss L., Infante E. F.: Finite-Time Stability under Perturbing forces and on Product Spaces, IEEE Transaction on Automatic Control, Vol. 12, 1967, pp. 54-59

[3] Angelo H. D.: Linear Time-Varying Systems: Analysis and Synthesis, Allyn and Bacon, Boston, 1970

[4] Amato F., Ariola M., Dorato P.: State Feedback Stabilization Over a FiniteTime Interval of Linear Systems Subject to Norm Bounded Parametric Uncertainties, Proc. of the $36^{\text {th }}$ Allerton Conference, Monticello, Sept. 2325, 1998, pp. 499-505

[5] Amato F., Ariola M., Dorato P.: Finite-Time Control of Linear Systems Subject to Parametric Uncertainties and Disturbances, Automatica, Vol. 37 No. 9, 2001, pp. 1459-1463

[6] Amato F., Ariola M., Cosentino C., Abdallah C. T., Dorato P.: Necessary and Sufficient Conditions for Finite-Time Stability of Linear Systems, Proc. of American Control Conference, Denver, Colorado, June 2003, pp. 4452-4456

[7] Amato F., Ariola M., Dorato P.: Finite-Time Stabilization via Dynamic Output Feedback, Automatica, Vol. 42, 2006, pp. 337-342

[8] Moulay E., Perruquetti W.: Finite-Time Stability and Stabilization of a Class of Continuous Systems, Journal of Mathematical Analysis and Applications, Vol. 323, 2006, pp. 1430-1443

[9] Ming Q., Shen Y.: Finite-Time Hळ Control for Linear Continuous System with Norm-bounded Disturbance, Communications in Nonlinear Science and Numerical Simulation, Vol. 14, 2009, pp. 1043-1049 
[10] G. Garcia, S. Tarbouriech, J. Bernussou, Finite-Time Stabilization of Linear Time-Varying Continuous Systems, IEEE Transaction on Automatic Control, Vol. 54, 2009, pp. 364-369

[11] Li P., Zheng Z.: Global Finite-Time Stabilization of Planar Nonlinear Systems with Disturbance, Asian Journal of Control, Vol. 14, No. 3, 2012, pp. $851-858$

[12] Debeljkovic D. Lj., Nenadic Z. Lj., Koruga Dj., Milinkovic S. A., Jovanovic M. B.: On Practical Stability of Time-Delay Systems: New Results, Proc. $2^{\text {nd }}$ ASCC97, Seoul, Korea, July 22-25, 1997, pp. 543-545

[13] Nenadic Z. Lj., Debeljkovic D. Lj., Milinkovic S. A.: On Practical Stability of Time Delay Systems, Proc. American Control Conference, Albuquerque, NM USA, 1997, pp. 3235-3235

[14] Lazarevic M. P., Debeljkovic D. Lj.: Finite Time Stability Analysis of Linear Autonomous Fractional Order Systems with Delayed State, Asian Journal of Control, Vol. 7, No. 4, 2005, pp. 440-447

[15] Lazarevic M. P., Debeljkovic D. Lj., Nenadic Z. Lj., Milinkovic S. A.: Finite-Time Stability of Delayed Systems, IMA Journal of Mathematical Control and Information, Vol. 17, No. 2, 2000, pp. 101-109

[16] Debeljkovic D. Lj., Lazarevic M. P., Koruga Dj., Milinkovic S. A., Jovanovic M. B.: Further Results on the Stability of Linear Nonautonomous Systems with Delayed State Defined Over Finite Time Interval, Proc. American Control Conference, Chicago, IL, USA, 2000, pp. 1450-1451

[17] Debeljkovic D. Lj., Buzurovic I.M., Nestorovic T., Popov D.: On Finite and Practical Stability of Time Delayed Systems: Lyapunov-Krassovski Approach: Delay Dependent Criteria, Proc. the $23^{\text {rd }}$ IEEE Chinese Control and Decision Conference CCDC, Mianyang, China, March 23-25, 2011, pp. 331-337

[18] Debeljkovic D. Lj., Stojanovic S. B., Jovanovic A.: Further Results on Finite Time and Practical Stability of Continuous Time Delay Systems, FME Transactions, Vol. 41, 2013, pp. 241-249

[19] Stojanovic S. B., Debeljkovic D. LJ.: Delay-Dependent Stability Analysis for Discrete-Time Systems with Time Varying State Delay, Chemical Industry \& Chemical Engineering Quarterly, Vol. 17 No. 4, 2011, pp. 497504

[20] Stojanovic S. B., Debeljkovic D. LJ., Dimitrijevic N.: Finite-Time Stability of Discrete-Time Systems with Time-Varying Delay, Chemical Industry and Chemical Engineering Quarterly, Vol. 18, No 4/I, 2012, pp. 525-533

[21] Stojanovic S. B., Debeljkovic D. Lj., Antic D. S.: Finite Time Stability and Stabilization of Linear Time Delay Systems, Facta Universitatis, Series Automatic Control and Robotics, Vol. 11, No. 1, 2012, pp. 25-36 
[22] Stojanovic S. B., Debeljkovic D. LJ., Antic D. S.: Robust Finite-Time Stability and Stabilization of Linear Uncertain Time-Delay Systems, Asian Journal of Control, Vol. 16, No. 2, 2013, DOI: 10.1002/asjc.689, pp. 1-7

[23] Lee T. N., Diant S.: Stability of Time-Delay Systems, IEEE Transactions on Automatic Control, Vol. 26, No. 4, 1981, pp. 951-953 